\title{
Fusion of Yeast Protoplasts Induced by Polyethylene Glycol
}

\author{
By A. SVOBODA \\ Department of Biology, Medical Faculty, J.E. Purkyně University, \\ 66243 Brno, Czechoslovakia
}

(Received 26 June 1978)

\begin{abstract}
Protoplasts, prepared from auxotrophic strains of Saccharomyces cerevisiae, Schizosaccharomyces pombe and Hansenula wingei, were mixed to give intraspecific complementary combinations. Polyethylene glycol (PEG) was added to induce agglutination and fusion. Some of the fused products grew on the surface of solid minimal medium forming large vacuolated bodies. Others reverted to hybrid cells when embedded in solid minimal regeneration medium. The cytological and preliminary genetical analyses suggest a synkaryon formation and integration of genetic markers from parental strains. The frequency of intrageneric fusions assessed from the number of protoplasts growing on the surface of minimal agar was estimated to be 1 to $3 \%$, while the frequency of hybrid colony formation in regeneration medium was less than $1 \%$.
\end{abstract}

\section{INTRODUCTION}

Protoplasts of fungi are useful models for cytological studies. They can be readily prepared, subjected to various treatments and cultivated. Under defined conditions they regenerate their walls within several hours and revert to normal cells (Nečas, 1971). The differentiation of the whole organism involves only the reversion of the regenerated protoplast into a normal cell; species-specific morphogenesis is already renewed by the first, second or third generations (Svoboda \& Nečas, 1974). For these reasons, protoplasts can serve as unique tools for fusion experiments. Successful fusions have already been reported between fungal protoplasts (Ferenczy et al., 1975; Anné \& Peberdy, 1975, 1976) and between yeast protoplasts (Sipiczki \& Ferenczy, 1977; van Solingen \& van der Plaat, 1977; Yamamoto \& Fukui, 1977).

Recently (Svoboda, 1976) I studied the possibility of fusion of yeast protoplasts derived from opposite mating-type cells. Sex-specific fusion occurred only after the wall had regenerated, indicating that mechanisms similar to those operating in intact cells are involved. Here I report the formation of artificial hybrids, between yeast auxotrophs, induced by PEG (Kao \& Michayluk, 1974) and a simple method for the detection of developing hybrid protoplasts.

\section{METHODS}

Organisms and growth conditions. Haploid strains with detectable biochemical markers were used. Saccharomyces cerevisiae strains 9 his $\alpha$ and $5 \operatorname{trp}$ a were obtained from Dr M. Opekarová, Institute of Microbiology, Prague, strain rec291 leu trp1-1 ura4 met4 tyr7 $\propto$ from Dr R. K. Mortimer, University of California, Berkeley, and strains $3901 \mathrm{C}$ his $\propto$ and 8282 ade $\alpha$ from Dr V. Kováčová, Komenský University, Bratislava. Schizosaccharomyces pombe strains leuI-32 $h^{-}$and ade6-250 $h^{-}$were obtained from Dr U. Leupold, University of Bern, and Hansenula wingei strains 5 cyh lys and 21 ade his were from Dr M. Crandall, University of Kentucky, Lexington.

All strains were maintained on wort agar slants. The cultures were grown in liquid wort medium at $28^{\circ} \mathrm{C}$ and before conversion to protoplasts they were cultivated in minimal medium (Leupold, 1955) for 
$2 \mathrm{~h}$. The protoplasts were cultivated in similar medium supplemented with $0.7 \mathrm{M}$-mannitol and $2 \%(\mathrm{w} / \mathrm{v})$ Difco agar (solid minimal medium).

Protoplast preparation and fusion. Protoplasts were prepared by the routine method using snail enzyme (Eddy \& Williamson, 1959). Cells were harvested in the exponential phase of growth, washed twice with distilled water and incubated in $0.01 \mathrm{M}$-mercaptoethanol for $30 \mathrm{~min}$ at $28{ }^{\circ} \mathrm{C}$. Saccharomyces cerevisiae cells were then suspended in a solution containing $0.7 \mathrm{M}$-mannitol, $1 \%(\mathrm{w} / \mathrm{v})$ lyophilized snail enzyme and $0 \cdot 15 \mathrm{M}$-citrate/phosphate buffer ( $\mathrm{pH} 5 \cdot 4$ ). Schizosaccharomyces pombe cells were suspended in a similar solution but with a lower $(0.6 \mathrm{M})$ mannitol concentration. For $H$. wingei cells, the buffered mannitol was replaced by $0.8 \mathrm{M}^{-\mathrm{MgSO}_{4}}$. Fresh protoplasts were washed with non-buffered $0.7 \mathrm{M}$-mannitol. After washing, protoplasts (about $10^{6}$ of each auxotroph) were mixed in an appropriate combination (Table 1) and centrifuged. The pellet was resuspended in $1 \mathrm{ml}$ of $30 \%(\mathrm{w} / \mathrm{v})$ polyethylene glycol (PEG; mol. wt 4000; Lachema, Brno, Czechoslovakia) containing $0.01 \mathrm{M}-\mathrm{CaCl}_{2}$. After incubation for $20 \mathrm{~min}$ at $37^{\circ} \mathrm{C}$ the suspension was slowly diluted with minimal medium containing $0.7 \mathrm{M}$-mannitol, and then washed twice with similar medium.

Growth and regeneration of fusion products. PEG-treated and control protoplasts were diluted in stabilized minimal medium, streaked by means of a capillary on a minimal agar-coated microscope coverslip, and then incubated in a moist chamber. The chamber provides excellent conditions for prolonged cultivation of protoplasts. The preparations were incubated at $28{ }^{\circ} \mathrm{C}$ and observed microscopically at regular intervals.

To observe regeneration of the wall and reversion to cells, the pellet of mixed protoplasts was embedded in $2 \%(\mathrm{w} / \mathrm{v})$ agar minimal medium in Petri dishes. The number of reverting protoplasts was determined microscopically. A group of at least ten cells around the regenerated protoplast was taken as indicating reversion.

Staining of nuclei. Nuclei in cells and protoplasts were counted in Feulgen-stained preparations.

\section{RESULTS}

\section{Growth and reversion of untreated protoplasts}

Both $S$. cerevisiae and $H$. wingei protoplasts require gel media for wall regeneration and reversion to cells. On the surface of agar or in liquid media they only grow and do not revert, forming an incomplete fibrillar wall (Nečas, 1971). However, protoplasts of Sch. pombe may revert under these conditions (Svoboda, 1966, 1967).

When spread on to the surface of solid minimal medium the protoplasts of all $S$. cerevisiae and $H$. wingei auxotrophs, either singly or in complementary mixtures (Table 1), failed to grow or grew to a limited extent only (Fig. 1). The slight increase in volume was apparently due to osmotic enlargement of the vacuole. Some protoplasts of Sch. pombe regenerated the walls but did not develop further.

Within solid minimal medium the auxotrophic protoplasts retained their spherical shapes (Fig. 2), but no reversion was observed in an inoculum of about $10^{4}$ protoplasts. In similar experiments with normal cells no colonies arose on minimal medium plates indicating a good stability of auxotrophic markers. With Sch. pombe, some revertants appeared within $2 \mathrm{~d}$ of cultivation as groups of one or two cells around the regenerated protoplasts. No visible colonies were found when an inoculum of more than $10^{4}$ protoplasts was used.

\section{Growth and reversion of PEG-treated protoplasts}

The addition of PEG to the protoplast suspension resulted in intensive agglutination which led to formation of large aggregates (Fig. 3). The number of protoplasts in the aggregates depended mainly on the density of the pellet: the more diluted the pellet, the less protoplasts in the clumps. During incubation at $37^{\circ} \mathrm{C}$ the aggregated protoplasts were stable without any visible lysis. On subsequent dilution with stabilized medium, the clumps were disrupted into single protoplasts among which some larger bodies still persisted. In these treated and washed protoplasts one to five nuclei were revealed by Feulgen staining (Fig. 4), while controls (untreated protoplasts) were regularly uni-nucleate. Evaluation of samples of PEG-treated and stained protoplasts showed up to $10 \%$ of bi- and multinucleate bodies. 

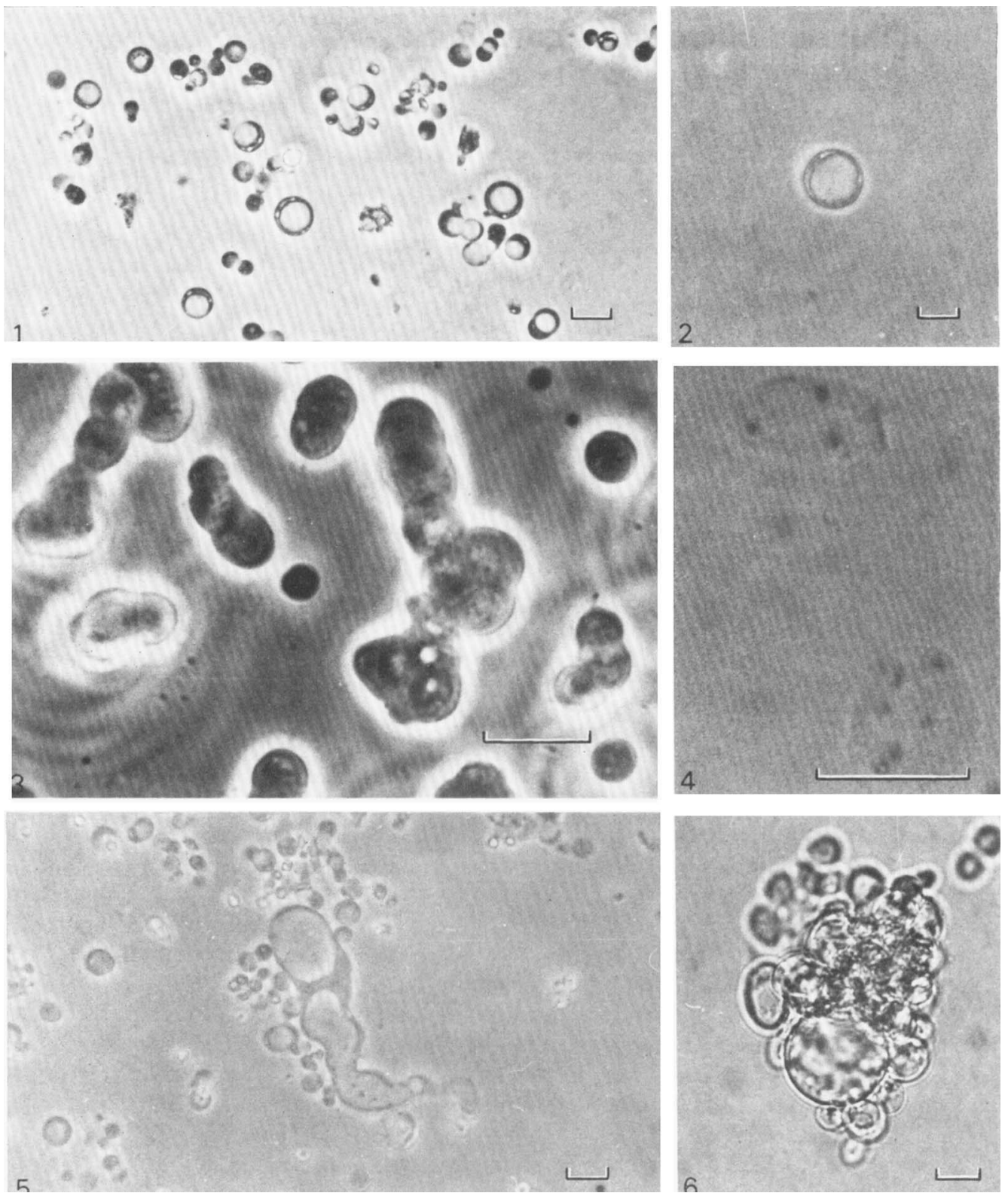

All bar markers represent $10 \mu \mathrm{m}$.

Fig. 1. Mixture of $S$. cerevisiae $291 \alpha+9 \alpha$ protoplasts grown for $24 \mathrm{~h}$ on the surface of solid minimal medium.

Fig. 2. One of the protoplasts from the same mixture grown for $24 \mathrm{~h}$ in solid minimal medium. Note the regenerated wall on the protoplast.

Fig. 3. Agglutination of $S$. cerevisiae $291 \alpha+9 \alpha$ protoplasts induced with $30 \%$ (w/v) PEG. Phase contrast microscopy.

Fig. 4. Nuclei in the treated and washed protoplasts as revealed after Feulgen staining

Fig. 5. PEG-treated mixture of $S$. cerevisiae $291 \alpha+9 \alpha$ protoplasts grown for $24 \mathrm{~h}$ on the surface of solid minimal medium. Note one growing vacuolated protoplast; the others are non-growing.

Fig. 6. Reverting protoplast from a PEG-treated mixture of S. cerevisiae $291 \alpha+9 \alpha$ protoplasts after $24 \mathrm{~h}$ incubation in solid minimal medium. Normal cells arise by budding from the regenerated protoplast. 
Table 1. Efficiency of protoplast fusion after treatment with $30 \%(w / v) P E G$

Fusion treatment was carried out as described in Methods. After washing free from PEG, the protoplasts were transferred on to minimal agar film or into minimal agar medium. The preparations were incubated at $28{ }^{\circ} \mathrm{C}$ and after $24 \mathrm{~h}$ the numbers of growing or reverting protoplasts were determined microscopically. The efficiency of fusion was expressed as a percentage of growing or reverting protoplasts from an inoculum of about $10^{4}$ protoplasts. The same mixtures of complementing protoplasts but untreated with PEG were used as controls.

\begin{tabular}{|c|c|c|c|c|}
\hline \multirow[b]{2}{*}{ Strains hybridized } & \multicolumn{2}{|c|}{$\begin{array}{c}\text { Growing protoplasts on } \\
\text { the surface of minimal } \\
\text { medium }(\%)\end{array}$} & \multicolumn{2}{|c|}{$\begin{array}{l}\text { Reverting protoplasts } \\
\text { in minimal } \\
\text { medium }(\%)\end{array}$} \\
\hline & Control & PEG & Control & PEG \\
\hline $\begin{array}{l}\text { Saccharomyces cerevisiae } \\
9 \text { his } \alpha+5 \text { trp a } \\
9 \text { his } \alpha+\text { rec } 291 \text { leu trp ura met tyr } \alpha \\
3901 \mathrm{C} \text { his } \alpha+5 \text { trp a } \\
8282 \text { ade } \alpha+9 \text { his } \alpha\end{array}$ & $\begin{array}{l}0 \\
0 \\
0 \\
0\end{array}$ & $\begin{array}{l}2 \cdot 7 \\
3 \cdot 2 \\
1 \cdot 3 \\
2 \cdot 1\end{array}$ & $\begin{array}{l}0 \\
0 \\
0 \\
0\end{array}$ & $\begin{array}{l}0 \cdot 1 \\
0 \cdot 1 \\
0 \cdot 08 \\
0 \cdot 1\end{array}$ \\
\hline $\begin{array}{l}\text { Schizosaccharomyces pombe } \\
\quad \text { leu } h^{-}+\text {ade } h^{-}\end{array}$ & 0 & $0 \cdot 5^{*}$ & 0 & 0.09 \\
\hline $\begin{array}{l}\text { Hansenula wingei } \\
5 \text { cyh lys }+21 \text { ade his }\end{array}$ & 0 & 0.01 & 0 & $-\dagger$ \\
\hline
\end{tabular}

For hybridization experiments, the suspension of mixed protoplasts (Table 1) was diluted so that aggregates of two to four protoplasts were formed after PEG addition.

When the PEG-treated and washed protoplast suspensions were seeded on to minimal agar films, some of them grew into large vacuolated formations (Fig. 5). It should be stressed that this was active growth with a progressive increase in the cytoplasmic volume. The frequency of growing protoplasts varied from one experiment to another and typical results are shown in Table 1. Similar results were found when the protoplasts were grown in liquid medium but under these conditions most lysed after 8 to $16 \mathrm{~h}$. In Sch. pombe auxotrophic combinations, growth of about $0.5 \%$ of the protoplasts was observed and occasionally some reversion occurred (one to three microcolonies per slide preparation).

The growing protoplasts were found not only in the aggregates but also singly, without any contact with other bodies. On the surface of solid minimal medium lysis of the protoplasts occurred only rarely, so the lysed material could hardly supplement the medium with substances that were lacking. These results, together with those showing the stability of auxotrophic markers, suggest that the growing protoplasts originate from successfully fused complementary protoplasts.

When the PEG-treated and washed protoplasts were embedded into solid minimal medium, some of them increased in volume, regenerated walls and reverted to cells (Table 1). Microcolonies of normal cells were formed in 12 to $16 \mathrm{~h}$ (Fig. 6). With further cultivation the colonies became visible and the cells could be transferred on to fresh minimal medium. Here again, these cells can be regarded as revertants of protoplasts which have arisen by fusion, as confirmed by a series of control experiments. As well as the controls already mentioned, these included: cultivation of PEG-treated non-complementing protoplast mixtures, cultivation of PEG-treated complementing protoplasts after lysis with distilled water and cultivation of PEG-treated cells in complementary combinations.

Other PEG concentrations ( 20 to $50 \%$ ), PEG solutions with $\mathrm{pH}$ values ranging from $5 \cdot 4$ to $10 \cdot 5$, temperatures of less than $37^{\circ} \mathrm{C}$ and incubation times greater than 20 min generally gave lower yields of prototrophic protoplasts than the procedure described in Methods. 

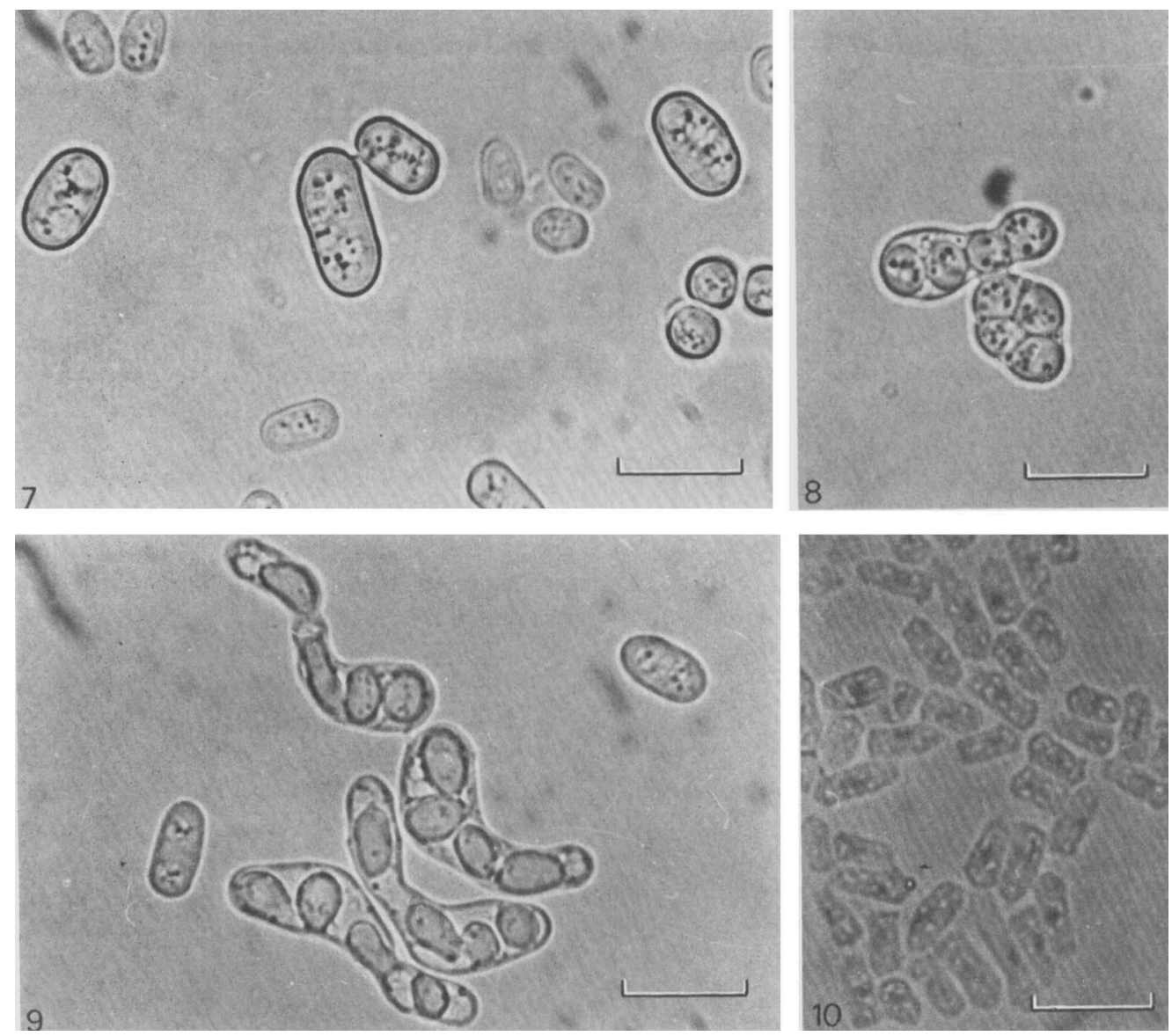

All bar markers represent $10 \mu \mathrm{m}$.

Fig. 7. Large hybrid cells of Sch. pombe leu $h^{-}+a d e h^{-}$, prepared by fusion, and their parent forms (smaller cells).

Fig. 8. Sporulating asymmetric zygotes prepared by crossing S. cerevisiae $(291 \alpha+9 \alpha) \times 5 a$.

Fig. 9. Spores in Sch. pombe zygotes $\left(l e u h^{-}+a d e h^{-}\right) \times h i s h^{+}$. Note the apparent asymmetry of the zygotes.

Fig. 10. Feulgen staining of hybrid cells Sch. pombe leu $h^{-}+$ade $h^{-}$. All the cells contain one nucleus only.

\section{Analysis of fusion products}

Hybrid cells were regularly larger than the parent strains (Fig. 7) and in their size they resembled diploid cells. The reverted $S$. cerevisiae cells originating from fusion of $\alpha+a$ protoplasts were typical diploids capable of sporulation. The cells from $\alpha+\alpha$ combinations were of $\alpha$ mating-type, incapable of sporulating and showed a haploid type of budding. Hybrids $291 \alpha+9 \alpha$ were flocculent like the parent strain $9 \alpha$ and could conjugate with strain $5 a$. Hybrid (larger) and haploid (smaller) cells formed asymmetric zygotes. The zygotes and cells arising from them sporulated, showing one to six spored asci with the maximum of four spores in an ascus (Fig. 8). Orientation genetical analyses using mitotic segregation in hybrids $291 \alpha+9 \alpha$, and random spore analysis in hybrids $(291 \alpha+9 \alpha) \times 5 a$, revealed that auxotrophic colonies requiring leucine, uracil, methionine, histidine, histidine plus uracil and tryptophan were segregated. No quantitative screening of segregants was made, however.

Hybrid cells originating from the fusion of Sch. pombe $\mathrm{h}^{-}+\mathrm{h}^{-}$protoplasts were of $\mathrm{h}^{-}$ 
mating-type and non-sporulating. They could conjugate with $\mathrm{h}^{+}$cells giving asymmetric zygotes with four spores (Fig. 9), but zygotes with six spores were also present. No detailed analysis of spores was performed.

The hybrid cells reverted from the fused protoplasts did not lose their prototrophic character after several passages on minimal media. They contained only one nucleus, as revealed by Feulgen staining (Fig. 10).

\section{DISCUSSION}

Artificially induced fusion has been demonstrated in a number of animal cells and plant and bacterial protoplasts (Harris \& Watkins, 1965; Power et al., 1970; Fodor \& Alföldi, 1976). It was reasonable to expect that yeast protoplasts could also be fused without any difficulties. The results :obtained here, like those reported recently (Sipiczki \& Ferenczy, 1977; van Solingen \& van der Plaat, 1977; Yamamoto \& Fukui, 1977), confirmed this assumption. The appearance of prototrophic growing and reverting protoplasts from mixtures of complementary auxotrophs is believed to result from protoplast fusion. Control experiments showed that cross-feeding, enrichment of the medium by components from disintegrated protoplasts and eventual reverse mutations can be excluded as possible causes of protoplast growth and reversion.

The frequencies of fusion as determined by hybrid colony formation were low but comparable with results obtained in mould protoplasts (Anné \& Peberdy, 1975, 1976). Very high frequencies of fusion, however, have been reported (Ferenczy et al., 1975). The low yield of resultant hybrids does not correspond with the observation of about $10 \%$ of biand multi-nucleate protoplasts after PEG treatment. This apparent discrepancy could be caused by disruption of some fused protoplasts during washing from PEG and, mainly, by insufficient embedding of protoplasts in gel which, at least in budding yeast, is the most important requirement for successful regeneration of the cell wall (Nečas, 1971). For comparison, not more than $10 \%$ of wild-type S. cerevisiae protoplasts reverted and produced colonies under the same cultivation conditions. It should also be mentioned that not all microcolonies manifest themselves into visible colonies and this may explain the relatively higher yield of hybrids in comparison with other published results (Sipiczki \& Ferenczy, 1977; van Solingen \& van der Plaat, 1977; Yamamoto \& Fukui, 1977).

The estimation of fusion rate based on the frequency of growing protoplasts on the surface of minimal agar media proved more reliable than that based on colony counts, although in the former case - except when Sch. pombe was studied - no cells were recovered. One can suggest that the growth of hybrid protoplasts is induced when the necessary genetic information is somehow introduced into a common cytoplasm, while regeneration of the cell wall and reversion to normal cells requires entire genome integration. In spite of these limitations the technique of cultivation of hybrid protoplasts on the surface of agar media can be recommended, at least for orientation experiments, as a test of whether fusions have occurred.

The finding of only a low frequency of growing hybrid protoplasts of $H$. wingei was not analysed further and its cause remained unclear. Due to persistence of some non-converted cells in protoplast preparations, which give rise to prototrophic colonies by conjugation, one cannot determine the exact number of reverted hybrid protoplasts, even when they are present.

These cytological and genetical studies have demonstrated that the integration of both parental genes into one synkaryon occurs in hybrid cells. This has also been demonstrated more precisely by Sipiczki \& Ferenczy (1977) in the case of Sch. pombe protoplasts.

The low yield of prototrophic regenerated protoplasts has not allowed a more detailed study of their morphology and morphogenesis which is our main objective. The final result obtained in this study was the production of large cells which were possibly diploid. How- 
ever, the regenerated protoplasts are likely to segregate not only diploids, but also dikaryons, polykaryons, polyploids or some parental forms. These problems can only be solved if the fusion frequency and the efficiency of reversion are improved.

The author wishes to thank Professor O. Nečas, Professor E. C. Cocking and Dr J. F. Peberdy for their criticism of the manuscript. The technical assistance of V. Janischová is gratefully acknowledged.

\section{REFERENCES}

Anné, J. \& Peberdy, J. F. (1975). Conditions for induced fusion of fungal protoplasts in polyethylene glycol solutions. Archives of Microbiology 105, 201-205.

ANNÉ, J. \& PEBERdy, J. F. (1976). Induced fusion of fungal protoplasts following treatment with polyethylene glycol. Journal of General Microbiology 92, 413-417.

Eddy, A. A. \& Williamson, D. H. (1959). Formation of aberrant cell walls and of spores by the growing yeast protoplasts. Nature, London 183, 1101-1104.

Ferenczy, L., Kevei, F. \& Szegedi, M. (1975). High frequency fusion of fungal protoplasts. Experimentia 31, 1028-1030.

Fodor, K. \& AlFöldi, L. (1976). Fusion of protoplasts of Bacillus megaterium. Proceedings of the National Academy of Sciences of the United States of America 73, 2147-2150.

Harris, H. \& Watkins, J. F. (1965). Hybrid cells derived from mouse and man: artificial heterokaryons of mammalian cells from different species. Nature, London 205, 640-646.

KaO, K. N. \& Michayluk, M. R. (1974). A method for high-frequency intergeneric fusion of plant protoplasts. Planta 115, 355-367.

LEUPOLD, U. (1955). Versuche zur genetischen Klassifizierung adeninabhängiger Mutanten von Schizosaccharomyces pombe. Archiv der Julius Klaus-Stiftung für Vererbungsforschung, Sozialanthropologie und Rassenhygiene 30, 506-516.
NeČAs, O. (1971). Cell wall synthesis in yeast protoplasts. Bacteriological Reviews 35, 149-170.

Power, J. B., Cummins, S. E. \& Cocking, E. C. (1970). Fusion of isolated plant protoplasts. Nature, London 225, 1016-1018.

SiPICZKI, M. \& Ferenczy, L. (1977). Protoplast fusion of Schizosaccharomyces pombe auxotrophic mutants of identical mating type. Molecular and General Genetics 157, 77-83.

van Solingen, P. \& Van der PlaAt, J. B. (1977). Fusion of yeast spheroplasts. Journal of Bacterio$\log y$ 130, 946-947.

Svoboda, A. (1966). Regeneration of yeast protoplasts in agar gels. Experimental Cell Research 44, 640-642.

SvobodA, A. (1967). Regeneration ability of protoplasts of different yeast species. In Symposium on Yeast Protoplasts, pp. 31-35. Edited by R. Müller. Berlin: Akademie-Verlag.

Svoboda, A. (1976). Mating reaction in yeast protoplasts. Archives of Microbiology 110, 313-318.

Svoboda, A. \& NeČas, O. (1974). Morphogenesis during protoplast reversion in dimorphic yeast. In Proceedings of the Fourth International Symposium on Yeast, p. D9. Edited by H. Klaushofer \& U. B. Sleyter. Wien: Hochschule für Bodenkultur.

Yамамото, M. \& Fukui, S. (1977). Fusion of yeast protoplasts. Agricultural and Biological Chemistry 41, 1829-1830. 